\title{
Impact of COVID-19 prevention measures on Clostridioides difficile infections in a regional acute care hospital
}

\author{
SIMONA SIPOS $^{1 *}$, CRISTIAN VLAD $^{1 *}$, RADU PREJBEANU $^{2,3}$, HORIA HARAGUS $^{2}$, \\ DALIBORCA VLAD ${ }^{1}$, HORIA CRISTIAN ${ }^{4}$, CRISTIAN DUMITRASCU $^{5}$,

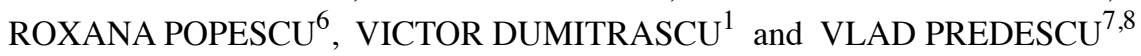

\begin{abstract}
Departments of ${ }^{1}$ Pharmacology and ${ }^{2}$ Orthopedics and Trauma, 'Victor Babes' University of Medicine and Pharmacy of Timisoara, 300041 Timisoara; ${ }^{3}$ Department of Orthopedics and Trauma, Premiere Hospital, 300643 Timisoara; Departments of ${ }^{4}$ Surgery II, ${ }^{5}$ Cardiovascular Surgery and ${ }^{6}$ Cell and Molecular Biology, 'Victor Babes' University of Medicine and Pharmacy of Timisoara, 300041 Timisoara; ${ }^{7}$ Department of Orthopedics and Trauma, 'Carol Davila' University of Medicine and Pharmacy, 050474 Bucharest;

${ }^{8}$ Department of Orthopedics and Trauma, 'Ponderas' Academic Hospital, 014142 Bucharest, Romania
\end{abstract}

Received May 17, 2021; Accepted June 16, 2021

DOI: $10.3892 / \mathrm{etm} .2021 .10649$

\begin{abstract}
Clostridioides difficile (C. difficile) is a common cause of nosocomial diarrhea. The multi-modal infection control strategies designed to contain the COVID-19 pandemic have had an unintended positive effect on other hospital-acquired infections. The aim of the present study was to analyze the impact of the COVID-19 prevention measures on healthcare-associated $C$. difficile infections in a large regional acute care center. Electronic databases were reviewed from the start of the pandemic (March) up to November 2020. Average values from the same months from 2019 and 2018 were used as controls. Using the ICD-10 discharge coding, 65 C.difficile cases per 25,124 patients were identified in 2020 compared to 151/43,126 from the 2018 and 2019 averages $(\mathrm{P}=0.0484)$. The $C$. difficile cases were found to be decreased after the implementation of COVID-19 infection control strategies compared to previous years, despite an increase in antibiotic use. Subset analysis during lockdown showed a clear decrease but the difference was not statistically significant. For the months of recovery after lockdown, the number of cases was comparable to previous years.
\end{abstract}

Correspondence to: Professor Radu Prejbeanu or Dr Horia Haragus, Department of Orthopedics and Trauma, 'Victor Babes' University of Medicine and Pharmacy of Timisoara, 2 Eftimie Murgu Square, 300041 Timisoara, Romania

E-mail: raduprejbeanu@gmail.com

E-mail: horia.haragus@umft.ro

${ }^{*}$ Contributed equally

Key words: Clostridium difficile, coronavirus infection, delivery of health care, hospitals, pandemics

\section{Introduction}

Clostridioides difficile ( $C$. difficile) is a problematic infection in hospitalized patients. It is one of the most common causes of both nosocomial and community-acquired diarrhea, with a reported prevalence of 13 cases per 1,000 patients in the US (1-4). C. difficile colonizes the large intestine in up to $8 \%$ of admitted patients. Spores are resistant to heat, acid and antibiotics, are present on surfaces and are transmitted via the hand-mouth route. $C$. difficile produces 2 toxins (A and $\mathrm{B}$ ) that cause colitis in susceptible hosts. Clinical manifestations range from asymptomatic to severe diarrhea, toxic megacolon and even death $(1,5)$.

Multiple factors lead to this disease, the most important and controllable of which appear to be exposure to spores from contaminated hands, excessive use of large spectrum antibiotics and length of hospitalization (1-5). Antimicrobial stewardship programs aimed to reduce the use of broad-spectrum antibiotics such as third and fourth generation cephalosporines, fluoroquinolones, macrolides, ampicillin and amoxicillin. $C$. difficile spores are virtually ubiquitous in healthcare facilities. Hand hygiene with soap and water, use of gloves, increased use of single use/disposable items, surface and terminal patient room disinfection have all been proven useful in containing the spread of $C$. difficile diarrhea as well as other nosocomial pathogens (1-5).

The COVID-19 (coronavirus disease 2019) pandemic has imposed significant restrictions and has brought major changes in the way hospitals manage patients (6-11). In Romania, the first case of COVID-19 was confirmed at the end of February, 2020. In an effort to stop the spread of the virus, a state of emergency was declared on the 16th of March and lasted until the 14th of May, 2020. In the following months, several infection control measures were implemented to reduce community transmission and prepare for the second wave. There was a generalized recommendation to wear face masks, limit the duration of interpersonal contact, disinfect 
surfaces, impose frequent hand hygiene with alcohol-based rubs and soap and water, use gloves, designate routes and limit unnecessary patient movement, reduce hospital length of stay, decrease patient density, isolate patients as much as possible, adhere to strict no touch-packaged meals, limit outside visitors, and increase the terminal disinfection of rooms (6-11).

These multi-modal infection control strategies designed to contain the COVID-19 pandemic have had an unintended positive effect on other hospital-acquired infections. It has been reported that these measures led to a significant decrease in cases of influenza, enterovirus, pneumonia MRSA (methicillin-resistant Staphylococcus aureus) or central-line-associated bloodstream infection. The same trend has been observed for hospital acquired $C$. difficile infections. However, these reports included either only COVID-19-positive patients or were limited to the lockdown period (12-17). Therefore, the hypothesis was that this trend may continue in the months after the lockdown has ended.

The aim of the present study was to analyze the impact of the COVID-19 prevention measures on healthcare-associated C. difficile infections in the 'Pius Brinzeu' Emergency County Hospital from Timisoara, Romania.

\section{Materials and methods}

We performed a retrospective electronic database analysis for hospital-acquired $C$. difficile infections in the tertiary-care teaching hospital 'Pius Brinzeu' Emergency County Hospital from Timisoara, Romania. The period from the start of the pandemic (March) up to (and including) November 2020 was reviewed. The average numbers of $C$. difficile cases per month from March to November of the previous 2 years (2018 and 2019) were used as controls.

$C$. difficile infections were determined by searching for ICD-10 (10th revision of the International Statistical Classification of Diseases and Related Health Problems) code A04.7 (Enterocolitis due to $C$. difficile) at discharge.

Antibiotic use for 2020 was checked and compared to the 2018 and 2019 monthly averages. The 7 most used antibiotics (by commercial name) were identified each month in each department. These were then counted as total doses of antibiotics and compared relative to total monthly number of inpatients and hospital bed days.

The same data extraction methods were applied to the tested 2020 period as well as controls (2018 and 2019). Descriptive statistics were prepared using Microsoft Excel (Microsoft). Results are presented as the number of cases per 100,000 inpatients and bed days, respectively. Continuous data were analyzed using the unpaired t-test. Categorical data were compared using the Fisher's exact test or Chi-square with Yates correction. Analysis was performed using GraphPad QuickCalcs (GraphPad Software, Inc.). A two-tailed $\mathrm{P}<0.05$ was considered as indicative of a statistically significant difference.

The study was conducted according to the guidelines of the Declaration of Helsinki and approved by the Institutional Review Board: 'Pius Brinzeu' Emergency County Hospital Ethics Committee for Scientific Research (no. 214/2020). Written informed consent was obtained from all the patients.

\section{Results}

In the reviewed period (March-November, 2018-2020), there were 111,743 patients admitted to the hospital accounting for a total of 476,003 inpatient bed days.

From March to November 2020, 65 C. difficile infections out of 25,124 patients were identified. This was significantly less than the 2018 and 2019 averages used as controls: $151 / 43,126, \mathrm{P}=0.0484$. Per 100,000 hospitalized patients and bed days there were 265.5 and 70.5 cases in 2020 and 354.5 and 80.8 from 2018 and 2019 averages, respectively. The results are presented in Tables I-III.

A comparison of only the months of the lockdown (March-May), the difference was not statistically significant $(\mathrm{P}=0.1103)$. Per 100,000 hospitalized patients there were 306 cases in 2020 and 469 from the 2018 and 2019 averages, respectively. For a comparison of only the months after the lockdown (June-November) and excluding August for being an outlier, the difference was not statistically significant $(\mathrm{P}=0.9562)$. Per 100,000 hospitalized patients there were 282 and 291 cases, respectively.

In 2020 there were more antibiotics used compared to the averages of the previous 2 years. The difference was statistically significant when compared relative to monthly inpatients $(\mathrm{P}=0.0007 ; 95 \% \mathrm{CI},-2.35$ to -0.76$)$ and bed days $(\mathrm{P}=0.0001$, $95 \% \mathrm{CI},-0.75$ to -0.32$)$.

\section{Discussion}

The results of the present study showed a decrease in C.difficile infections after the implementation of COVID-19 control strategies compared to previous years, despite an increase in antibiotic use. This effect was maintained up to 9 months.

Bentivegna et al also observed a significant reduction in the number of $C$. difficile cases after the onset of the COVID-19 pandemic. Their analysis included the period from March until June but excluded intensive care units and pediatric wards (15). Wee et al reported that the cumulative incidence of healthcare-associated respiratory viral infection decreased from 9.69 cases per 10,000 patient days to 0.83 from February to August 2020. On the other hand, $C$. difficile cases only decreased from 3.65 to 3.47 per 10,000 patient-days compared to the pre-pandemic and the difference was not statistically significant (12). During the first wave (March-May) of the COVID-19 pandemic, Ponce-Alonso et al noted a $70 \%$ reduction in $C$. difficile cases in COVID-19 RT-PCR (reverse transcription-polymerase chain reaction-confirmed) patients, in spite of a slightly higher antibiotic consumption (14).

Clostridioides difficile (formerly known as Clostridium difficile or $C$. difficile) is a gram-positive, anaerobic, spore-forming bacillus. Viable spores have been found on surfaces, bed sheets, common instruments, hands and clothing of medical personnel $(1,2,5)$. The A and B exotoxins may lead to death of the cells lining the intestinal mucosa, loss of the gut barrier function and colitis. The organism itself is not invasive and remains in the intestine. A healthy fecal microbiota is protective against colonization with toxigenic strains of C. difficile. This microenvironment is severely disturbed when administering broad-spectrum antibiotics. 
Table I. Clostridioides difficile cases/100,000 hospitalized patients (no.).

\begin{tabular}{lcccccccccc}
\hline & \multicolumn{10}{c}{ Month } \\
\cline { 2 - 9 } Time period & March & April & May & June & July & August & September & October & November & Averages \\
\hline 2020 & 262 & 335 & 344 & 406 & 197 & 0 & 216 & 246 & 380 & 265.5 \\
Controls & 389 & 632 & 361 & 280 & 256 & 375 & 397 & 235 & 261 & 354.5 \\
\hline
\end{tabular}

Controls, monthly averages for 2018 and 2019 based on ICM-10 coding.

Table II. Clostridioides difficile cases/100,000 hospital bed days (cases).

\begin{tabular}{lrrrrrrrrrr}
\hline & \multicolumn{10}{c}{ Month } \\
\cline { 2 - 9 } Time period & March & April & May & June & July & August & September & October & November & Averages \\
\hline 2020 & 61 & 92 & 107 & 106 & 49 & 0 & 52 & 63 & 102 & 70.5 \\
Controls & 88 & 138 & 82 & 64 & 60 & 87 & 89 & 54 & 60 & 80.8 \\
\hline
\end{tabular}

Controls, monthly averages of 2018 and 2019 based on ICM-10 coding.

Table III. Monthly Clostridioides difficile cases (no.).

\begin{tabular}{lcccc}
\hline Month & Group & All & C.difficile cases & P-value \\
\hline March & 2020 & 3,042 & 8 & \\
& Controls & 4,988 & 20 & 0.34 \\
April & 2020 & 1,487 & 5 & \\
& Controls & 4,318 & 28 & 0.23 \\
May & 2020 & 2,315 & 8 & \\
& Controls & 4,963 & 19 & 0.9999 \\
June & 2020 & 2,941 & 12 & \\
& Controls & 4,797 & 14 & 0.42 \\
July & 2020 & 3,538 & 7 & \\
& Controls & 5,062 & 13 & 0.65 \\
August & 2020 & 2,717 & 0 & \\
& Controls & 4,108 & 16 & 0.0004 \\
September & 2020 & 3,226 & 7 & \\
& Controls & 4,638 & 19 & 0.16 \\
October & 2020 & 3,243 & 8 & \\
& Controls & 5,290 & 13 & 0.9999 \\
November & 2020 & 2,615 & 10 & \\
& Controls & 4,956 & 13 & 0.38 \\
\hline
\end{tabular}

Controls, monthly average Clostridioides difficile infections of 2018 and 2019 based on ICM-10 coding.

Diagnosis is generally made by enzyme immunoassay testing for the toxins in the stool. DNA-based tests can detect toxigenic strains and provide higher sensitivity and specificity. However, these tests are less common, may also detect infections that are not clinically meaningful and thus lead to an increase in false-positive results $(1,2,5)$. Cultures are not routinely used. For hospitalized patients, testing is recommended only in cases with diarrhea, defined as 3 consecutive unformed stools. Incidence of $C$. difficile diarrhea is higher in elderly patients $(1,18-20)$. The same patient may present another episode of $C$. difficile infection. There is a controversial distinction between recurrence and reinfection in most published reports. This stems from definitions, heterogeneity and performance of diagnostic tests and variable time intervals. Nevertheless, the risk of a second episode is estimated to be between 10 and $50 \%(1,5)$.

Restricted antibiotic use has been proven to effectively limit $C$. difficile infections, but applicability in the clinical setting is extremely difficult. In the European Union, the use of broad-spectrum antibiotics is high. Prescription is higher in the ambulatory setting. An increase in antibiotic use can also lead to an increase of asymptomatic hosts which produce more spore contamination (1-5,18-27). Both oral metronidazole and vancomycin appear to have the same efficacy for mild and moderate forms. Vancomycin is considered better for severe disease and certain situations can also be addressed by fecal microbiota transplantation $(1,2,18)$. Treatment outcome is evaluated clinically since false-positive toxin tests may persist after treatment with favorable outcome.

COVID-19 control strategies have also led to large-scale use of daily and terminal disinfection of patient rooms and high-touch surfaces with bleach, hospital environmental cleaning, decrease in patient density, visitor restrictions and universal use of face masks (6-13). These methods in conjunction may be responsible for the reduction of hospital-acquired $C$. difficile diarrhea, because of the important overlap with previously proven $C$. difficile hygiene prevention measures (22-27).

Healthcare-associated infections present a serious risk and problem worldwide. Many other pathogens are responsible for increased morbidity, mortality and economic burden (1,2,24-26). A study by Gentili et al found an average 
prevalence of $5.24 \%$ of healthcare-associated infections in a teaching acute care hospital over 6 years. Respiratory tract infections were the most common, followed by surgical site, urinary tract, bloodstream infections, $C$. difficile and central nervous system infections (25). A study on the causes of diarrhea found norovirus to be the most common pathogen, followed by bacteria, parasites and coinfections (26).

As the COVID-19 pandemic continues to evolve, measures for spread containment have become the new daily routine. Face masks are now mandatory in all medical care facilities for both personnel and patients. All person to person direct contacts are limited to those that are absolutely essential. For patients admitted to hospital, visits of family members have been restricted. Unnecessary patient movement has been limited and managed using specially designated routes. For the purpose of reducing inpatient density, there is a strong emphasis for ambulatory treatment of both medical and surgical pathologies and a reduction in hospital length of stay. This creates the premises to isolate patients as much as possible. Both medical personnel and patients follow strict hand hygiene rules. Before and after interpersonal contact, the hands are rubbed with alcohol-based disinfectants, and all procedures are performed using disposable gloves and gowns. High-touch surfaces and floors are frequently disinfected. Terminal disinfection is applied as much as possible for all rooms. Meals are distributed exclusively in no-touch packaging (6-11). Wee et al found that with such multi-modal control strategies there was also a decline in healthcare-associated respiratory viral infections, central-line-associated bloodstream infections and all multi-drug resistance organisms: MRSA (methicillin-resistant Staphylococcus aureus) infections, vancomycin-resistant Enterococcus, carbapenemase-producing Enterobacteriaceae as well as $C$. difficile infections (12). Chiu et al analyzed the impact of wearing masks, hand hygiene, and social distancing on influenza, enterovirus, and all-cause pneumonia during the COVID-19 response and found a marked decrease for all cases of respiratory and seasonal viral infections (13). Another aspect weighing in on the efficacy of COVID-19 containment strategies is the improved compliance and generalized implementation $(6,8,10-14,27)$. Although most of these measures were in practice for many years, this is the first time they were rigorously followed and applied in all levels of society.

There are several limitations to the present study, beginning with the errors inherent to the retrospective design. Reoccurrences were not included, data pertaining to the cases prior to hospital admittance and after discharge, symptom severity, treatment and final outcome were not collected. The overall significant reduction in the number of cases was influenced by an outlier, i.e., the month of August with no $C$. difficile cases. The increased antibiotic use may have been linked to COVID-19 patients. However, data of the current study on antibiotic consumption was analyzed only by approximation of doses of the most common substances. In addition, a definitive cause for increased antibiotic use in 2020 was not found.

The present study included patients from a regional acute care hospital from the onset of the pandemic and into the second wave with community transmission. The $C$. difficile cases decreased after the implementation of COVID-19 infection control strategies compared to previous years, along with an increase in antibiotic use. Subset analysis during lockdown showed a clear decrease but the difference was not statistically significant. For the months of recovery after lockdown, the number of cases was comparable to previous years.

\section{Acknowledgements}

Bogdan Deleanu for helping with project management and Marius Sucilea with data analysis.

\section{Funding}

The APC was partially funded by the 'Victor Babes' University of Medicine and Pharmacy of Timisoara, Timisoara, Romania.

\section{Availability of data and materials}

The datasets used and/or analyzed during the current study are available from the corresponding author on reasonable request.

\section{Authors' contributions}

SS, RaP, DV, VD, HH and VP were involved in the conception of the study and the interpretation of the data. SS, CV, HC, $\mathrm{CD}, \mathrm{HH}$ and RoP contributed to the acquisition of the data and performed statistical analysis. SS, CV, HC, CD, HH and RoP wrote the manuscript. SS, RaP, DV, VD, HH and VP revised the manuscript for important intellectual content. All authors read and approved the final manuscript.

\section{Ethics approval and consent to participate}

The study was conducted according to the guidelines of the Declaration of Helsinki and approved by the Institutional Review Board: 'Pius Brinzeu' Emergency County Hospital Ethics Committee for Scientific Research (no 214/2020). All patients provided written informed consent.

\section{Patient consent to participate}

Not applicable.

\section{Competing interests}

The authors declare that they have no competing interests.

\section{References}

1. Leffler DA and Lamont JT: Clostridium difficile infection. N Engl J Med 372: 1539-1548, 2015.

2. Louh IK, Greendyke WG, Hermann EA, Davidson KW, Falzon L, Vawdrey DK, Shaffer JA, Calfee DP, Furuya EY and Ting HH: Clostridium difficile infection in acute care hospitals: Systematic review and best practices for prevention. Infect Control Hosp Epidemiol 38: 476-482, 2017.

3. Jenkins TC, Knepper BC, Shihadeh K, Haas MK, Sabel AL, Steele AW, Wilson ML, Price CS, Burman WJ and Mehler PS: Long-term outcomes of an antimicrobial stewardship program implemented in a hospital with low baseline antibiotic use. Infect Control Hosp Epidemiol 36: 664-672, 2015.

4. Kazakova SV, Baggs J, McDonald LC, Yi SH, Hatfield KM, Guh A, Reddy SC and Jernigan JA: Association between antibiotic use and hospital-onset clostridioides difficile infection in US acute care hospitals, 2006-2012: An ecologic analysis. Clin Infect Dis 70: 11-18, 2020. 
5. Petrosillo $\mathrm{N}$ and Cataldo MA: Clostridioides difficile infection: A room for multifaceted interventions. J Clin Med 9: 4114, 2020.

6. Scarlat MM and Mavrogenis AF: Orthopaedic Surgery during COVID pandemic and consequent changes in our professional environment. Int Orthop 44: 1607-1609, 2020.

7. Docea AO, Tsatsakis A, Albulescu D, Cristea O, Zlatian O, Vinceti M, Moschos SA, Tsoukalas D, Goumenou M, Drakoulis N, et al: A new threat from an old enemy: Re-emergence of coronavirus (Review). Int J Mol Med 45: 1631-1643, 2020.

8. Kenanidis E, Anagnostis P, Arvaniti K, Potoupnis ME and Tsiridis E: Organizing an orthopaedic department during COVID-19 pandemic to mitigate in-hospital transmission: Experience from Greece. Cureus 12: e8676, 2020.

9. Alexandru L, Haragus H, Deleanu B, Timar B, Poenaru DV and Vlad DC: Haematology panel biomarkers for humeral, femoral, and tibial diaphyseal fractures. Int Orthop 43: 1567-1572, 2019.

10. Calina D, Docea AO, Petrakis D, Egorov AM, Ishmukhametov AA, Gabibov AG, Shtilman MI, Kostoff R, Carvalho F, Vinceti M, et al: Towards effective COVID-19 vaccines: Updates, perspectives and challenges (Review). Int J Mol Med 46: 3-16, 2020.

11. Contini C, Caselli E, Martini F, Maritati M, Torreggiani E, Seraceni S, Vesce F, Perri P, Rizzo L and Tognon $M$ COVID-19 is a multifaceted challenging pandemic which needs urgent public health interventions. Microorganisms 8: $1228,2020$.

12. Wee LEI, Conceicao EP, Tan JY, Magesparan KD, Amin IBM Ismail BBS, Toh HX, Jin P, Zhang J, Wee EGL, et al: Unintended consequences of infection prevention and control measures during COVID-19 pandemic. Am J Infect Control 49: 469-477, 2021.

13. Chiu NC, Chi H, Tai YL, Peng CC, Tseng CY, Chen CC, Tan BF and Lin CY: Impact of wearing masks, hand hygiene, and social distancing on influenza, enterovirus, and All-cause pneumonia during the coronavirus pandemic: Retrospective National epidemiological surveillance study. J Med Internet Res 22: e21257, 2020.

14. Ponce-Alonso M, Sáez de la Fuente J, Rincón-Carlavilla A, Moreno-Nunez P, Martínez-García L, Escudero-Sánchez R, Pintor R, García-Fernández $\mathrm{S}$ and Cobo J: Impact of the coronavirus disease 2019 (COVID-19) pandemic on nosocomial Clostridioides difficile infection. Infect Control Hosp Epidemiol 42: 406-410, 2021

15. Bentivegna E, Alessio G, Spuntarelli V, Luciani M, Santino I, Simmaco M and Martelletti P: Impact of COVID-19 prevention measures on risk of health care-associated Clostridium difficile infection. Am J Infect Control 49: 640-642, 2021.

16. Granata G, Bartoloni A, Codeluppi M, Contadini I, Cristini F, Fantoni M, Ferraresi A, Fornabaio C, Grasselli S, Lagi F, et al: The burden of clostridioides difficile infection during the COVID-19 pandemic: A retrospective case-control study in Italian Hospitals (CloVid). J Clin Med 9: 3855, 2020.

17. Ochoa-Hein E, Rajme-López S, Rodríguez-Aldama JC, Huertas-Jiménez MA, Chávez-Ríos AR, de Paz-García R, Haro-Osnaya A, González-Colín KK, González-González R, González-Lara MF, et al: Substantial reduction of healthcare facility-onset Clostridioides difficile infection (HO-CDI) rates after conversion of a hospital for exclusive treatment of COVID-19 patients. Am J Infect Control 49: 966-968, 2021.
18. Teja B, Alibhai N, Rubenfeld GD, Taggart LR, Jivraj N, Hirji SA, O'Gara BP and Shaefi S: Prevalence of clostridioides difficile infection in critically Ill patients with extreme leukocytosis and diarrhea. Infect Dis Rep 13: 18-22, 2021.

19. Putot A, Astruc K, Barben J, Mihai AM, Nuss V, Bador J, Putot S, Dipanda M, Laborde C, Vovelle J, et al: Impact of a diagnosis-centered antibiotic stewardship on incident clostridioides difficile infections in older inpatients: An observational study. Antibiotics 9: 303, 2020.

20. Jachowicz E, Różańska A, Pobiega M, Topolski M and Wójkowska-Mach J: Consumption of antibiotics and epidemiology of clostridioides difficile in the European Union in 2016-opportunity for practical application of aggregate ECDC Data. Antibiotics (Basel) 9: 127, 2020.

21. Muntean D, Horhat FG, Bădițoiu L, Dumitrașcu V, Bagiu IC, Horhat DI, Cosnită DA, Krasta A, Dugăesescu D and Licker M: Multidrug-resistant gram-negative bacilli: A retrospective study of trends in a tertiary healthcare unit. Medicina (Kaunas) 54: 92, 2018.

22. Macașoi I, Pavel IZ, Moacă AE, Avram S, David VL, Coricovac D, Mioc A, Spandidos DA, Tsatsakis A, Şoica C, et al: Mechanistic investigations of antitumor activity of a Rhodamine B-oleanolic acid derivative bioconjugate. Oncol Rep 44: 1169-1183, 2020.

23. Vlad CS, Dumitrascu V, Vlad DC, Popescu R, Cimporescu A, Flangea C, Cheveresan A, Sipos S, Bareac B and Malita I: Effects of materials based on acrylic resines on inflammatory markers IL- 8 and TGF $\beta 1$ in gingival crevicular fluid. Mater Plast 55: 111-114, 2018.

24. Tudoran C, Tudoran M, Lazureanu VE, Marinescu AR, Pop GN, Pescariu AS, Enache A and Cut TG: Evidence of pulmonary hypertension after SARS-CoV-2 infection in subjects without previous significant cardiovascular pathology. J Clin Med 10: 199,2021

25. Gentili A, Di Pumpo M, La Milia DI, Vallone D, Vangi G, Corbo MI, Berloco F, Cambieri A, Damiani G, Ricciardi W and Laurenti P: A six-year point prevalence survey of healthcare-associated infections in an Italian teaching acute care hospital. Int J Environ Res Public Health 17: 7724, 2020.

26. Casillas-Vega N, Flores-Rodríguez F, Sotelo-Coronado I, Vera-García ME, García-Heredia A, Rivas-Estilla AM, Lozano-Sepúlveda SA, García S, Flores-Arechiga A and Heredia N: Norovirus is the most frequent cause of diarrhea in hospitalized patients in Monterrey, Mexico. Pathogens 9: 672, 2020.

27. Israel S, Harpaz K, Radvogin E, Schwartz C, Gross I, Mazeh H, Cohen MJ and Benenson S: Dramatically improved hand hygiene performance rates at time of coronavirus pandemic. Clin Microbiol Infect 26: 1566-1568, 2020.

This work is licensed under a Creative Commons Attribution-NonCommercial-NoDerivatives 4.0 International (CC BY-NC-ND 4.0) License. 